\title{
Strong-field-gated buildup of a Rydberg series
}

\author{
Veit Stooß, ${ }^{*}$ Paul Birk $\odot,{ }^{*}$ Alexander Blättermann, Maximilian Hartmann $\odot$, Gergana D. Borisova $\odot$, \\ Christian Ott $\odot$, and Thomas Pfeifer ${ }^{\dagger}$ \\ Max-Planck-Institut für Kernphysik, Saupfercheckweg 1, 69117 Heidelberg, Germany
}

(Received 15 May 2020; accepted 2 July 2020; published 18 August 2020)

\begin{abstract}
The femtosecond-timescale formation of a Fano resonance in doubly excited helium has been recently observed, enabling a time-domain view into two-electron correlation dynamics. Measuring the absorption spectral line shape, the buildup of the resonance is revealed by imposing a temporal gate on the dipole response using strong-field ionization. Here, we apply this approach to the Rydberg series of the doubly excited states in helium. This reveals the characteristic times of emergence of isolated two-electron resonances from the continuous single-ionization background absorption, as well as their time-dependent line-shape asymmetry and the time it takes to separate individual spectral absorption lines within the series. Furthermore, we time resolve the dynamics of the excited wave packet by reconstructing its dipole response in the time domain and thereby characterize the ionization gate to close as fast as only $1.2 \mathrm{fs}$. These results represent an approach to resolve on the femtosecond and attosecond timescale the strong-field-ionization dynamics of excited coherent wave packets.
\end{abstract}

DOI: 10.1103/PhysRevResearch.2.032041

\section{INTRODUCTION}

The measurement of photoabsorption and photoemission spectra of atoms in response to external stimuli reveals their quantized energy structure as well as the associated induced electron dynamics, which typically unfolds on the attosecond time scale. With the advent of attosecond science, the real-time investigation of ultrafast electron dynamics came within experimental reach. Using attosecond pulses, pumpprobe measurements have been realized in both photoelectron spectroscopy, e.g., the attosecond streak camera [1] and the reconstruction of attosecond beating by interference of twophoton transitions technique (RABBITT) [2-4] as well as attosecond transient absorption spectroscopy [5], and are used with great success to gain new insight into, e.g., (correlated) electron dynamics [6-8] or ultrafast decay channels during autoionization [9].

A typical spectral signature of autoionization is the Fano resonance [10]. Here, an excited state is degenerate with, and coupled to a continuum. This degeneracy leads to quantumpath interference and configuration interaction, which in turn results in an asymmetry of the spectroscopic absorption line shape. A crucial question here, and in general for spectral signatures of correlated electron dynamics, is how the interference between different quantum paths emerges and how long this process takes. This question has first been investigated in theory [11-16] and has recently been addressed also

\footnotetext{
*These authors contributed equally to this work.

${ }^{\dagger}$ Corresponding author: tpfeifer@mpi-hd.mpg.de
}

Published by the American Physical Society under the terms of the Creative Commons Attribution 4.0 International license. Further distribution of this work must maintain attribution to the author(s) and the published article's title, journal citation, and DOI. Open access publication funded by the Max Planck Society. experimentally for the helium atom $[17,18]$. The theoretically predicted emergence of a Fano resonance has been measured with both absorption spectroscopy [17] and photoelectron spectroscopy [18], yielding very good agreement of the respective experimental results with theory. In the absorption measurement, the formation of the Fano resonance has been resolved by imposing a time gate on the excited dipole response of the target by means of strong-field ionization by an intense few-cycle near-infrared (NIR) laser pulse. By varying its time delay to the extreme ultraviolet (XUV) excitation pulse, the time-dependent formation of the resonant Fano line shape can be directly probed from the measured XUV photoabsorption spectrum.

This strong-field-gating approach immediately leads to further questions. Considering that commonly used analytical models of strong-field ionization are typically based on the single active electron approximation [19,20], one key question in recent work is the role of the dynamics with two active electrons during the process of strong-field ionization [21-24]. Furthermore, in order to develop a more comprehensive timedomain understanding, one ideally should resolve the buildup dynamics not only for an isolated resonance, as has been done previously [17], but also for a coherently excited wave packet involving multiple two-electron excited states, e.g., for a Rydberg series.

In this paper, we use the time-gating approach developed in Ref. [17] to observe the formation of more complex resonance structures in helium. We experimentally measure the ultrafast buildup of a series [25,26] of multiple doubly excited states close to the $N=2$ ionization continuum of the excited helium ion $\mathrm{He}^{+^{*}}$, which manifests as a series of partly overlapping Fano resonances. We examine how long it takes for the Fano quantum-path interference and consequently for the spectral lines to emerge. We investigate their spectral separation over time in a set of closely spaced absorption lines and show how the measured buildup differs from a simplified analytical 
(a)
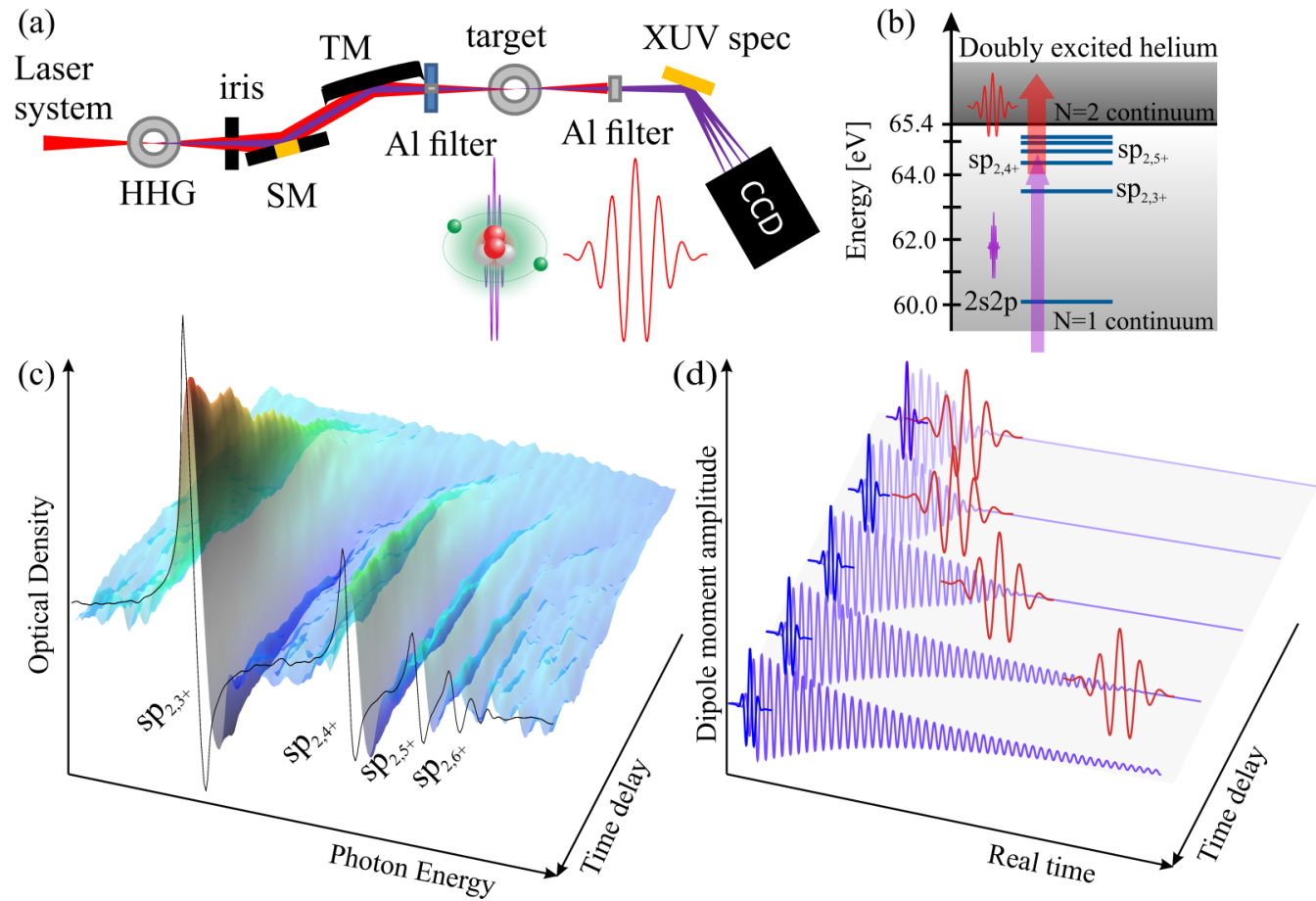

FIG. 1. Measurement scheme. (a) Schematic of the experimental setup. HHG: High-order harmonic generation in neon. SM: Split-mirror setup for setting the XUV-NIR time delay. TM: Toroidal focusing mirror. Target: Absorption in helium. XUV spec: XUV flat-field spectrometer. (b) Schematic energy-level diagram of the investigated doubly excited states. (c) Measured data of the buildup of a series of Fano resonances. (d) Illustration of the action of the time-gating technique on the dipole response in the time domain [17].

model calculation, where we treat each resonance independently and neglect any coherent wave-packet dynamics. We further quantify on the femtosecond timescale the strongfield-driven dynamics during the ionization of the coherent wave packet by reconstructing the strongly perturbed dipole response in the time domain from the measured absorption spectra [27].

\section{EXPERIMENT}

In order to reveal the formation and time-dependent depletion of the excited resonances close to the $N=2$ ionization threshold in helium, a measurement with combined high spectral and temporal resolution is necessary. The experiment has been performed with the setup schematically illustrated in Fig. 1(a), together with a method for in situ XUV reference measurements for attosecond transient absorption spectroscopy. The setup, which is further described in Ref. [28], allows for a sufficient spectral resolution and sensitivity to measure time-dependent changes in the absorption spectrum of several doubly excited states of the $s p_{2, \mathrm{n}+}\left({ }^{1} P^{\mathrm{o}}\right)$ series in helium [see Figs. 1(b) and 1(c)]. The XUV absorption spectra are recorded while scanning the time delay of an intense few-cycle NIR laser pulse with a central wavelength of $726 \mathrm{~nm}$. Based on the measured XUV spectra, the XUV pulse duration is estimated to be considerably shorter than the driving NIR pulse, consisting of a few-pulse attosecond pulse train with dominant contribution from a main pulse and weaker satellite pulses. As stated in Ref. [17] and illustrated in Fig. 1(d), the strong-field ionization during the interaction with the NIR pulse acts as a time-gate mechanism. The NIR pulse duration of $\approx 6$ fs hereby defines an upper limit to the closing time of the gate and needs to be shorter than the autoionization decay time of the XUV doubly excited states. For higher-lying doubly excited states in helium with an autoionization decay time on the order of $100 \mathrm{fs}$ or longer this condition is fulfilled. Furthermore, in order for the time gate to be viable, the NIR intensity needs to be higher than $10^{13} \mathrm{~W} / \mathrm{cm}^{2}$.

Figure 2(a) shows NIR-cycle-averaged data of the measured time delay scan for an NIR intensity of $1.4 \times$ $10^{13} \mathrm{~W} / \mathrm{cm}^{2}$. This averaging procedure suppresses line modifications due to resonant couplings (most dominantly observed across the $s p_{23+}$ and $s p_{24+}$ states), which are signatures of wave-packet dynamics with the $2 s 2 p$ state and are typically observed as fast modulations of the absorption signal along the time-delay axis. For the study of the formation of the Rydberg series only the region of positive time delay is considered, where the position of the XUV pulse marks time zero, and the NIR pulse arrives later. In the time-delay region around $\tau=+5 \mathrm{fs}$, i.e., shortly after the XUV-NIR pulse overlap, the resonance lines of the doubly excited states are strongly suppressed and broadened by the strong-field ionization. The NIR pulse duration and the overlap time are calibrated in situ by using the dipole-control model for lower NIR intensities as described in Ref. [29], which results in a full-width-at-half-maximum pulse duration of $5.7 \pm 0.1 \mathrm{fs}$. With the grazing-incidence spectrometer, providing an energy resolution of $E / \Delta E \geqslant 1500$, the states of the $s p_{2, \mathrm{n}+}$ Rydberg series are resolved up to the $s p_{2,8+}$ state. 

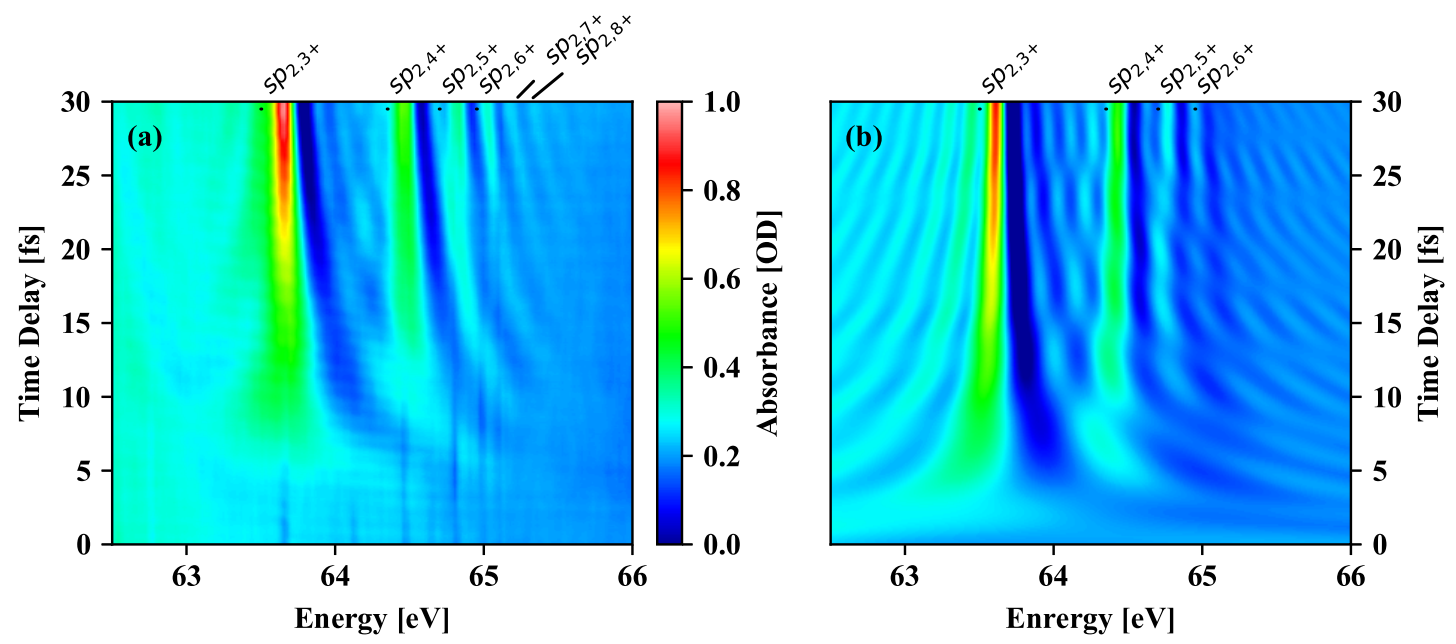

FIG. 2. Buildup measurement and analysis. (a) Time-delay scan of the observed buildup of the Rydberg series of doubly excited states in helium converging to the $N=2$ ionization threshold. Note that sub-NIR-cycle oscillations are strongly suppressed through the application of a sliding time-delay average over one NIR cycle of 2.4 fs. (b) Numerical calculations using the analytical dipole-control model [30], computing the evolution of the dipole response that is cut off instantaneously after a variable time delay $\tau$.

\section{DISCUSSION}

\section{A. Comparison of the buildup measurement with the dipole-control model}

Our first goal is to examine the formation and separation of partly overlapping resonances in time and study effects and changes in the buildup caused by the interaction of different electron configurations. The measured data are compared to simulated spectra, which use the analytical dipole-control model (DCM) [30], depicted in Fig. 2(b). In the framework of this model, an initially XUV-excited response at $t=0$ is instantaneously cut off after a variable time delay $\tau$, which simulates the complete depletion of the involved states due to strong-field ionization. For the simulation of the absorption spectra by the DCM the analytical formula

$$
S^{\mathrm{DCM}}(E, \tau)=c_{0}+\alpha E+\beta E^{2}+\gamma E^{3}+E \times \operatorname{Im}\left[\sum_{j=1}^{n} A_{j} \frac{1-e^{-\Gamma_{j} \tau /(2 \hbar)-i\left(E-E_{j}\right) \tau / \hbar}\left[1-A_{j}^{\mathrm{DCM}}(\tau) e^{\left.i \varphi_{j}^{\mathrm{DCM}}\right]}\right.}{\left(E-E_{j}\right)-i \Gamma_{j} / 2} e^{-i \varphi_{j}^{\mathrm{Fano}}}\right]
$$

is used, where $E$ is the XUV photon energy, $\hbar$ is the reduced Planck constant, and $A_{j}, E_{j}, \Gamma_{j}$, and $\varphi_{j}^{\text {Fano }}$ characterize the amplitude, energy position, linewidth, and asymmetry, respectively, of the individual resonances. $A_{j}^{\mathrm{DCM}}$ and $\varphi_{j}^{\mathrm{DCM}}$ describe the amplitude and phase modifications of the response due to the interaction with the NIR field. The parameters $c_{0}, \alpha, \beta$, and $\gamma$ are used to model the nonresonant background absorption. The only predetermined input for Eq. (1) is the width of the absorption lines, obtained from synchrotron measurements [26]. All other parameters which characterize the spectral lines $\left(A_{j}, E_{j}\right.$, and $\left.\varphi_{j}^{\text {Fano }}\right)$ are obtained from a fit to a measured reference spectrum for zero NIR intensity, with the constraints $A_{j}^{\mathrm{DCM}}=1$ and $\varphi_{j}^{\mathrm{DCM}}=0$. To account for the experimental finite spectrometer resolution, Eq. (1) is convolved $(*)$ with a Gaussian function $K(E)$. The width of this Gaussian is retrieved by a fit to measured data for zero NIR intensity. The full spectrum is given by

$$
S(E, \tau)=S^{\mathrm{DCM}}(E, \tau) * K(E) .
$$

The time-delay scan shown in Fig. 2(b) is obtained by evaluating Eq. (2) via setting $A_{j}^{\mathrm{DCM}}=1-\theta(\tau)$, i.e., full depletion during a Dirac- $\delta$-like NIR interaction at time $t=\tau$, and keeping $\varphi_{j}^{\mathrm{DCM}}=0$, where $\theta(t)$ denotes the Heaviside step function. In this model, all resonances are treated independently, incoherently summing over their individual contributions to the absorption spectra. This means coherent wavepacket dynamics or electron correlation effects are neglected.

Comparing the experimental data with the DCM, shown in Fig. 2, the buildup of the Rydberg series as well as characteristic hyperbolic structures originating from the rapid termination of the dipole response are both reproduced well by Eqs. (1) and (2). In addition, however, several key differences appear. First, the spectral lines in the experiment appear shifted towards higher photon energies at small time delays. This is likely due to the ac Stark effect or the ponderomotive shift above the $N=2$ ionization threshold, which are induced by the presence of the strong NIR pulse and most dominantly affect the early times during the buildup. This effect does not show up in the DCM as it is not included within the framework of this model with Dirac- $\delta$-like interactions. Furthermore, the abrupt truncation of the dipole response causes the hyperbolic sidebands to be overemphasized within the DCM. It essentially broadens the resonant absorption lines yielding the characteristic hyperbolic structure for every resonance over 

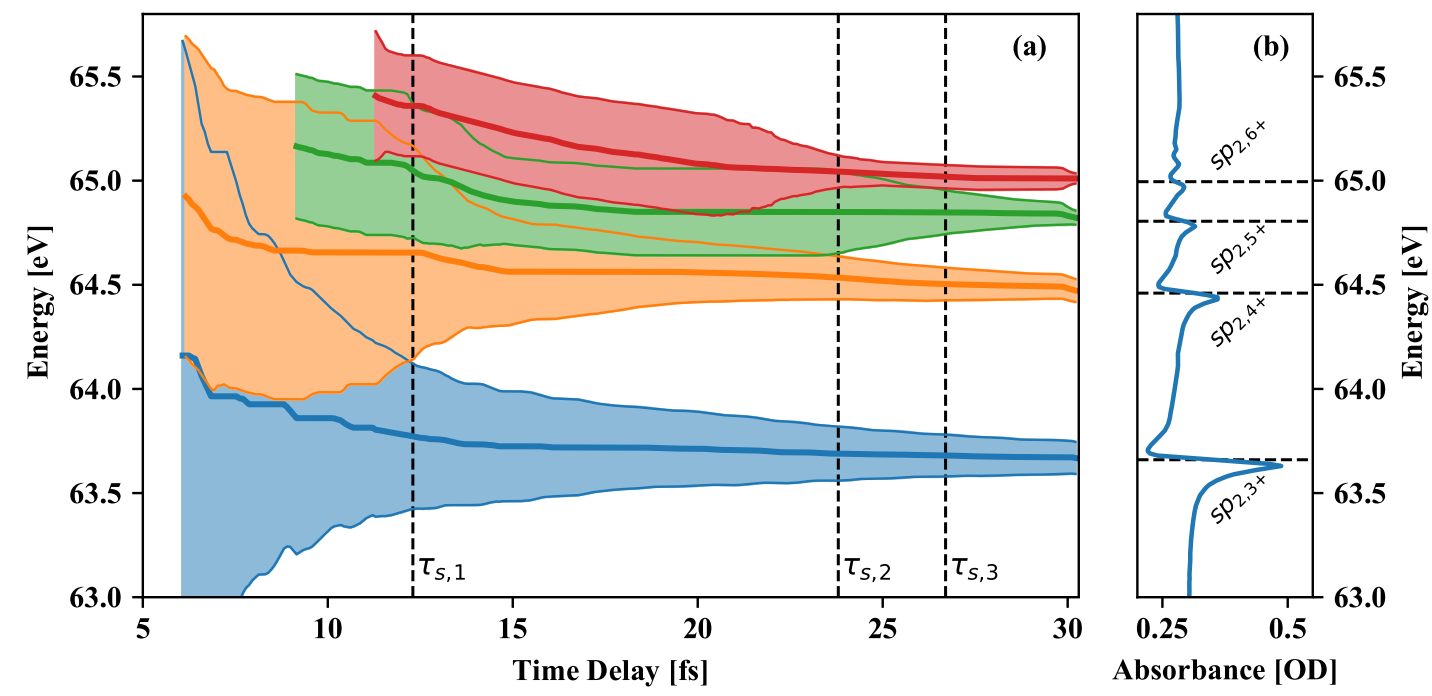

FIG. 3. Quantitative analysis of the emergence of the $s p_{2, \mathrm{n}+}$ Rydberg series in doubly excited helium. (a) Results of the fit algorithm. Solid lines indicate the resonance position $E_{\mathrm{j}}$; shaded areas indicate the resonance width $\Gamma_{\mathrm{j}}$. The times $\tau_{\mathrm{s}, \mathrm{i}}$ (vertical dashed lines) mark the delay when spectral lines are separable for the first time during the buildup scan. (b) Reference spectrum measured at $\tau \approx-12.5 \mathrm{fs}$.

a larger energy range. This also creates and emphasizes the XUV interference features which mask the appearance of individual resonances especially close to the $N=2$ ionization threshold. Thus, the difference in these oscillating hyperbolic sidebands results from the finite duration of the excited-state depletion during the interaction with the NIR pulse in the experiment. A more quantitative analysis of the intrapulse ionization dynamics will be presented further below, while first we extract the characteristic times of emergence of individual resonances and their time-delay-dependent asymmetry.

\section{B. Quantitative analysis of the buildup of the Rydberg series}

In order to examine the evolution of the amplitude, width, line-shape asymmetry, and separation of the individual but partly overlapping resonances within the experiment, we fit a series of modified Fano resonances to the absorption data, which incorporate an NIR-induced line-shape change. The fit function (see also Ref. [31]) is given by

$$
\begin{aligned}
S^{\mathrm{Fano}}(E)= & c_{0}+\alpha E+\beta E^{2}+\gamma E^{3} \\
& +\sum_{j=1}^{n} A_{j} \frac{\left(q_{0, j}^{2}+1\right)}{\left(q_{\mathrm{mod}, j}^{2}+1\right)}\left(\frac{\left(q_{\mathrm{mod}, j}+\varepsilon_{j}\right)^{2}}{\varepsilon_{j}^{2}+1}-1\right),
\end{aligned}
$$

where $E$ is the XUV photon energy. The amplitude $A_{j}$, the reduced resonance energy $\varepsilon_{j}=\left(E-E_{j}\right) /\left(\Gamma_{\mathrm{j}} / 2\right)$, the width $\Gamma_{j}$, and the asymmetry parameter $q_{0, j}$ characterize the individual unperturbed resonances while $q_{\bmod , j}$ gives the lineshape modification introduced by the NIR interaction. The parameters $c_{0}, \alpha, \beta$, and $\gamma$ describe again the nonresonant background absorption. This function is also convolved with the spectrometer resolution $K(E)$.

In Fig. 3(a) we present the energy positions and widths from the fits obtained for the different time delays. Here, the shaded areas around the resonance positions (traced by solid lines) indicate the width characterized by $\Gamma_{j}$. The respective fit results are plotted only for resonance amplitudes being greater than the spectral noise of the nonresonant background. This quantification clearly reveals that with increasing time delay all resonances become narrower and shift towards lower energies, approaching their unperturbed energy positions as they are less influenced by the NIR-induced ac Stark shift. In Fig. 3(b) we show a reference spectrum measured for negative time delay $\tau \approx-12.5 \mathrm{fs}$ when the NIR pulse has completely passed the target prior to the XUV excitation and thus does not affect the spectral lines.

Within this quantitative analysis, the spectral lines can be defined as being separate if they are further apart than the mean value of their widths, i.e., if the indicated widths around the resonance position in Fig. 3(a) do not overlap anymore. The time delay values $\tau_{s, i}$ in Fig. 3(a) (dashed vertical lines) indicate when the lines become separable. This can be well determined for the first three pairs of resonances $s p_{2,3+}-s p_{2,4+}\left(\tau_{\mathrm{s}, 1} \approx 12.3 \mathrm{fs}\right), s p_{2,4+}-s p_{2,5+}\left(\tau_{\mathrm{s}, 2} \approx\right.$ $23.8 \mathrm{fs})$, and $s p_{2,5+}-s p_{2,6+}\left(\tau_{\mathrm{s}, 3} \approx 26.7 \mathrm{fs}\right)$. We observe that the separation time is longer for state pairs with smaller energy differences. This can be intuitively explained as follows: the closer the resonances are in their energy positions, the longer the resonantly excited dipole moment needs to freely evolve in time. Only then separable absorption lines are generated. Furthermore, Fig. 3 reveals the time delays at which the respective spectral lines are discernible from the nonresonant continuum absorption background for the first time. The lines appear later the closer the resonances are located to the $N=2$ continuum. This can be explained by their increasingly closer proximity to neighboring states, their lower binding energy, as well as their smaller linewidth, which results in a lower amplitude, after taking into account the spectrometer resolution, and makes it harder for them to be discernible from the nonresonant background.

In Fig. 4 the time evolution of the line-shape modification is shown, which is expressed by the modified asymmetry 


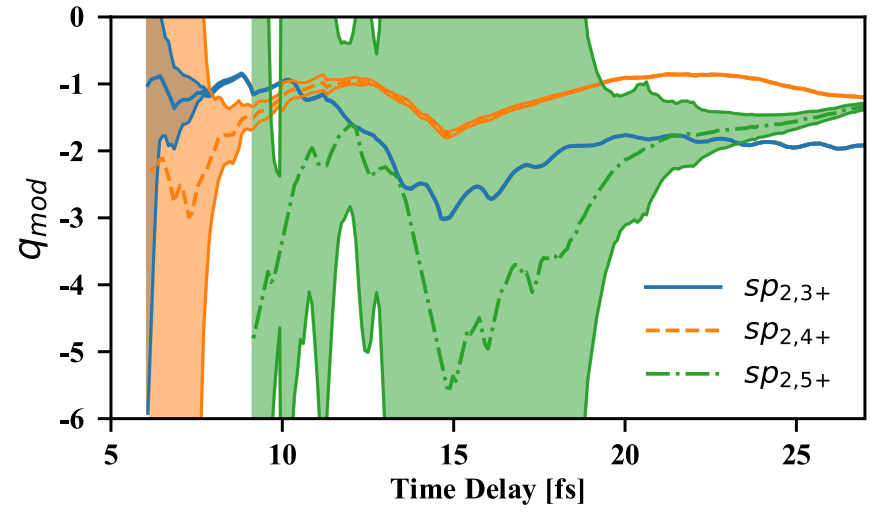

FIG. 4. Quantitative analysis of the modified $q$ parameter of the $s p_{2, \mathrm{n}+}$ Rydberg series in doubly excited helium with the shaded area indicating the standard fit error. The $q$ parameters show a common local minimum around time delay $15 \mathrm{fs}$, which might be connected to coherent wave-packet dynamics of the Rydberg series. For small time delays-shortly after XUV excitation - the amplitudes of the absorption lines are small leading to a large error in the determination of the modified $q$ parameter. As for larger delays the lines become more discernible from the background absorption, the error rapidly decreases.

parameter $q_{\text {mod }}$. This parameter captures the strength of the configuration interaction of the autoionizing state with the $\mathrm{He}^{+}$continuum and the XUV-induced dipole couplings to the ground state, which results in the Fano line shape [10]. Here we observe a modification of the line-shape asymmetry throughout the buildup of the coherently excited Rydberg series. In addition, the modified $q$ parameters show a common minimum at $15 \mathrm{fs}$, which may be related to coherent wave-packet dynamics, where the real-time reconstructed dipole moment (see Fig. 5 below) reveals a wave-packet beating on a comparable time scale. Further theoretical investigation with more elaborate $a b$ initio model simulations is, however, necessary to clearly resolve the underlying origin of the here observed time-dependent asymmetry changes for individual spectral lines within this coherently excited Rydberg wave packet of doubly excited states in helium.

\section{Gated wave-packet dynamics in the time domain}

In order to capture the dynamics that occur during the NIR strong-field interaction, we reconstruct the time-dependent dipole moment by Fourier transforming the absorption signal with a window on the wave packet with all Rydberg states starting from the $s p_{2,3+}$ in the energy region $[62.5 \mathrm{eV}$, $69.0 \mathrm{eV}$ ] (see Fig. 1), a method described in Ref. [27]. In Fig. 5(a) we show the dipole amplitude obtained by the realtime reconstruction applied to the experimentally measured absorption spectra for different time delays. For negative delays, the unperturbed beating of the wave packet along the real-time axis is observed, which is characteristic for the energy difference of the highly excited Rydberg states participating in the dynamics. At pulse overlap the dipole response is truncated in time starting at small negative time delays, and for later delays the modulations of the dipole amplitude recover following the NIR pulse.

Using the reconstructed amplitude of the dipole response, the position of the time gate as well as its finite duration imposed by the ionization during the NIR pulse can be quantified. For this purpose, a reference response $A_{d}(t)$ is multiplied with a time-gate function [see Eq. (4)]. This reference response is obtained by averaging over the reconstructed dipole amplitude in the time-delay range between -20 and -12 fs, i.e., a time-delay region when the NIR precedes the XUV excitation. The product is used to fit the reconstructed response data for positive time delays between 4 and $30 \mathrm{fs}$, i.e., the time-delay region when the NIR strongly affects the excited-state dynamics. We note that in both time-delay regions the NIR pulse does not overlap with the XUV pulse. In a first approximation, the time-gate function can be modeled
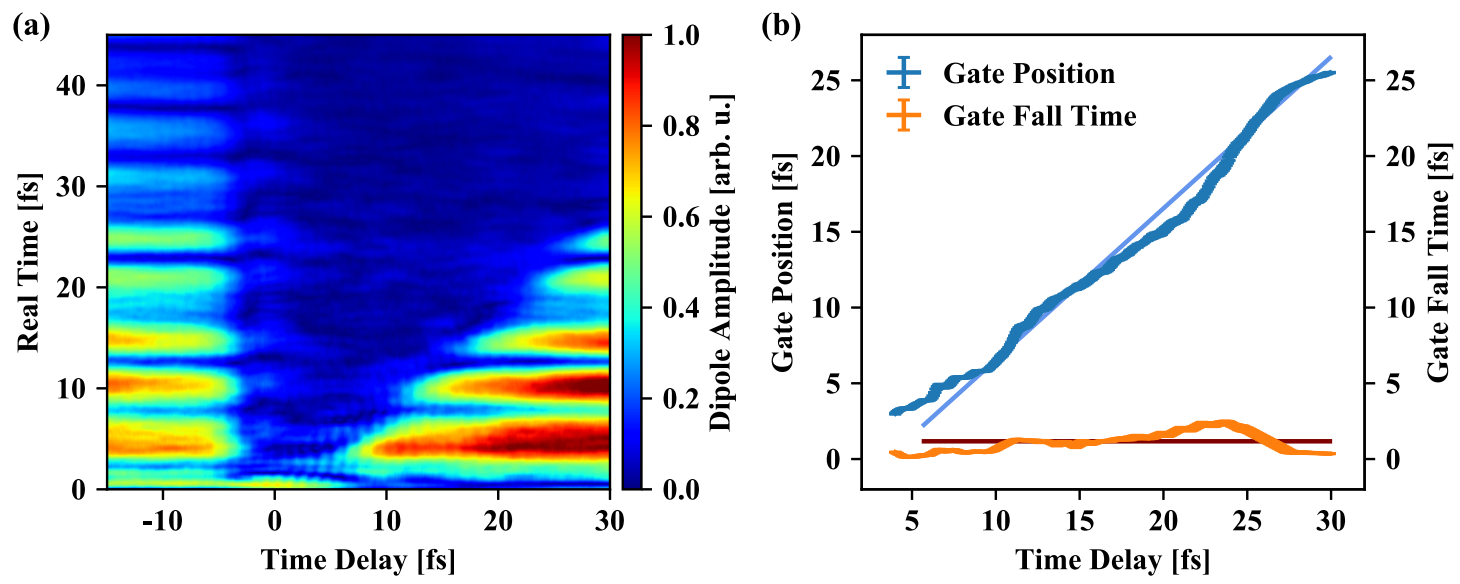

FIG. 5. Gated wave-packet dynamics in doubly excited helium. (a) Amplitude of the time-dependent dipole moment, reconstructed for a series of doubly excited states in helium starting from $s p_{2,3+}$. (b) Results of the time-gate fit to the reconstructed dipole moments [see Eq. (4)]. The blue and orange points give the gate position and fall time for a wave packet consisting of coherently excited Rydberg states. The line thickness indicates the statistical error over three separate time-delay scans with the same NIR intensity. Straight blue and red lines are a linear and constant-value fit to the experimentally extracted gate position and fall time, respectively. 
with a sigmoidal gate, which gives the fit function:

$$
R(t)=A_{d}(t)\left(\frac{1}{e^{\frac{t-t_{g}}{\Sigma}}+\frac{1}{A_{g}}}+\left(1-A_{g}\right)\right) .
$$

The free fit parameters in Eq. (4) are given by the gate fall time $\Sigma$, the gate position $t_{g}$, and the gate amplitude $A_{g}$. The latter accounts for a possible incomplete depletion for a realistic gate of NIR strong-field ionization. Despite this possibility, the fit results reveal an almost complete depletion with $0.9<$ $A_{g}<1$ for all time delays.

The orange and blue curves in Fig. 5(b) show the fitted fall time and position, respectively, taking the mean over three separate measurements with the same NIR intensity as used for the results shown in Sec. II. The thickness of the curves hereby quantifies the standard deviation. Both position and fall time show the expected behavior of the gate linearly following the NIR pulse with increasing time delay and constant fall time, respectively. By repeated fitting of the gate position or fall time, while fixing the other parameter at its expected behavior, we ascertained negligible crosstalk between the combined fit results. A fit of the thus obtained gate position with a linear function of slope 1 [light blue curve in Fig. 5(b)] results in an intercept at zero time delay at $t_{g}=-3.4 \mathrm{fs}$. We conclude that not the center but already the low-intensity leading edge of the pulse (3.4 fs before its maximum) is sufficiently strong to fully ionize the excited helium states and thereby gate the dynamics. A fit of the gate fall time with a time-delay-independent constant [straight red line in Fig. 5(b)] results in $\Sigma=1.2 \mathrm{fs,} \mathrm{which} \mathrm{is} \mathrm{much} \mathrm{shorter} \mathrm{than}$ the pulse duration of the NIR, revealing an extremely fast gate closure of the dipole dynamics through strong-field ionization. We further observe a slight deviation of the linear gate behavior, which might be connected to the beating dynamics of the coherently excited Rydberg wave packet. It may alter the strong-field ionization on a (sub-)femtosecond timescale. A more detailed investigation of this, where both the gate position and fall time can be clocked with subfemtosecond precision, might further clarify the role of dynamics of different coherent wave packets to the strong-field ionization process, which is, however, beyond the scope of the current paper.

\section{CONCLUSION}

The presented results show that the measurement of the buildup dynamics of complex spectral signatures consisting of multiple asymmetric Fano resonances can be achieved with the strong-field-induced time-gating technique. As a result, the overall buildup, the time-dependent separation, and line-shape-asymmetry modifications of multiple Fano resonances close to the $N=2$ ionization threshold in helium have been extracted quantitatively. Additionally, the time-domain observation of the dipole response revealed the ionization gate position and a short gate fall time of only 1.2 fs. This experiment and the retrieval of real-time attosecond dynamics in strong-field light-matter interaction directly from absorption spectra of complex spectral signatures represent a significant step towards high-precision quantum dynamics measurements within time-resolved spectroscopy. In the future, given a higher spectral resolution of the all-XUV-optical detection, the gating approach combined with time-domain observation introduced here could be used to reveal statespecific ionization dynamics close to a continuum threshold; many-body effects due to Rydberg interactions, which further influence the time-dependent buildup and evolution of highly excited states through interatomic interactions; as well as the quantification of the real-time evolution of bound-state electronic wave-packet dynamics in strong-field light-matter interaction in general.

\section{ACKNOWLEDGMENT}

We acknowledge funding from the European Research Council (Grant No. X-MuSiC-616783).
[1] J. Itatani, F. Quéré, G. L. Yudin, M. Y. Ivanov, F. Krausz, and P. B. Corkum, Phys. Rev. Lett. 88, 173903 (2002).

[2] H. G. Muller, Appl. Phys. B 74, s17 (2002).

[3] P. M. Paul, E. S. Toma, P. Breger, G. Mullot, F. Auge, P. Balcou, H. G. Muller, and P. Agostini, Science 292, 1689 (2001).

[4] M. Kotur, D. Guénot, Á. Jiménez-Galán, D. Kroon, E. W. Larsen, M. Louisy, S. Bengtsson, M. Miranda, J. Mauritsson, C. L. Arnold, S. E. Canton, M. Gisselbrecht, T. Carette, J. M. Dahlström, E. Lindroth, A. Maquet, L. Argenti, F. Martín, and A. L'Huillier, Nat. Commun. 7, 10566 (2016).

[5] E. Goulielmakis, Z.-H. Loh, A. Wirth, R. Santra, N. Rohringer, V. S. Yakovlev, S. Zherebtsov, T. Pfeifer, A. M. Azzeer, M. F. Kling, S. R. Leone, and F. Krausz, Nature (London) 466, 739 (2010).

[6] M. Ossiander, F. Siegrist, V. Shirvanyan, R. Pazourek, A. Sommer, T. Latka, A. Guggenmos, S. Nagele, J. Feist, J. Burgdörfer, R. Kienberger, and M. Schultze, Nat. Phys. 13, 280 (2017).
[7] M. Drescher, M. Hentschel, R. Kienberger, M. Uiberacker, V. Yakovlev, A. Scrinzi, T. Westerwalbesloh, U. Kleineberg, U. Heinzmann, and F. Krausz, Nature (London) 419, 803 (2002).

[8] M. Isinger, R. J. Squibb, D. Busto, S. Zhong, A. Harth, D. Kroon, S. Nandi, C. L. Arnold, M. Miranda, J. M. Dahlström, E. Lindroth, R. Feifel, M. Gisselbrecht, and A. L'Huillier, Science 358, 893 (2017).

[9] H. Wang, M. Chini, S. Chen, C.-H. Zhang, F. He, Y. Cheng, Y. Wu, U. Thumm, and Z. Chang, Phys. Rev. Lett. 105, 143002 (2010).

[10] U. Fano, Phys. Rev. 124, 1866 (1961).

[11] C. A. Nicolaides, T. Mercouris, and Y. Komninos, J. Phys. B 35, L271 (2002).

[12] T. Mercouris, Y. Komninos, and C. A. Nicolaides, Phys. Rev. A 69, 032502 (2004).

[13] M. Wickenhauser, J. Burgdörfer, F. Krausz, and M. Drescher, Phys. Rev. Lett. 94, 023002 (2005).

[14] T. Mercouris, Y. Komninos, and C. A. Nicolaides, Phys. Rev. A 75, 013407 (2007). 
[15] W. C. Chu and C. D. Lin, Phys. Rev. A 82, 053415 (2010).

[16] L. Argenti and E. Lindroth, Phys. Rev. Lett. 105, 053002 (2010).

[17] A. Kaldun, A. Blättermann, V. Stooß, S. Donsa, H. Wei, R. Pazourek, S. Nagele, C. Ott, C. D. Lin, J. Burgdörfer, and T. Pfeifer, Science 354, 738 (2016).

[18] V. Gruson, L. Barreau, Á. Jiménez-Galan, F. Risoud, J. Caillat, A. Maquet, B. Carré, F. Lepetit, J.-F. Hergott, T. Ruchon, L. Argenti, R. Taïeb, F. Martín, and P. Salières, Science 354, 734 (2016).

[19] L. V. Keldysh, Sov. Phys. JETP 20, 1307 (1965).

[20] M. V. Ammosov, N. B. Delone, and V. P. Krainov, Sov. Phys. JETP 64, 1191 (1986).

[21] P. Lambropoulos, P. Maragakis, and J. Zhang, Phys. Rep. 305, 203 (1998).

[22] A. N. Artemyev, L. S. Cederbaum, and P. V. Demekhin, Phys. Rev. A 95, 033402 (2017).

[23] W.-C. Jiang, S.-G. Chen, L.-Y. Peng, and J. Burgdörfer, Phys. Rev. Lett. 124, 043203 (2020).
[24] G. D. Borisova, V. Stooß, A. Dingeldey, A. Kaldun, T. Ding, P. Birk, M. Hartmann, T. Heldt, C. Ott, and T. Pfeifer, J. Phys. Commun. 4, 055012 (2019).

[25] H. D. Morgan and D. L. Ederer, Phys. Rev. A 29, 1901 (1984).

[26] M. Domke, K. Schulz, G. Remmers, G. Kaindl, and D. Wintgen, Phys. Rev. A 53, 1424 (1996).

[27] V. Stooß, S. M. Cavaletto, S. Donsa, A. Blättermann, P. Birk, C. H. Keitel, I. Březinová, J. Burgdörfer, C. Ott, and T. Pfeifer, Phys. Rev. Lett. 121, 173005 (2018).

[28] V. Stooß, M. Hartmann, P. Birk, G. D. Borisova, T. Ding, A. Blättermann, C. Ott, and T. Pfeifer, Rev. Sci. Instrum. 90, 053108 (2019).

[29] A. Blättermann, C. Ott, A. Kaldun, T. Ding, V. Stooß, M. Laux, M. Rebholz, and T. Pfeifer, Opt. Lett. 40, 3464 (2015).

[30] A. Blättermann, C. Ott, A. Kaldun, T. Ding, and T. Pfeifer, J. Phys. B 47, 124008 (2014).

[31] C. Ott, A. Kaldun, P. Raith, K. Meyer, M. Laux, J. Evers, C. H. Keitel, C. H. Greene, and T. Pfeifer, Science 340, 716 (2013). 